\title{
Entamoeba histolytica Phosphoserine aminotransferase (EhPSAT): insights into the structure-function relationship
}

\author{
Vibhor Mishra', Vahab Ali², Tomoyoshi Nozaki ${ }^{3}$, Vinod Bhakuni ${ }^{*}$
}

\begin{abstract}
Background: Presence of phosphorylated Serine biosynthesis pathway upstream to the de novo cysteine biosynthesis pathway makes PSAT a crucial enzyme. Besides this, phoshoserine produced by the enzyme can also be taken up directly by cysteine synthase as a substrate. PSAT is a PLP dependent enzyme where the cofactor serves as an epicenter for functional catalysis with the active site architecture playing crucial role in optimum function of the enzyme.

Findings: EhPSAT is a homodimer of molecular mass $86 \mathrm{kDa}$. To understand the structural modulations associated with $\mathrm{pH}$ dependent changes in functional activity of EhPSAT detailed biophysical studies were carried out. pH alterations had no significant effect on the secondary structure, cofactor orientation and oligomeric configuration of the enzyme however, $\mathrm{pH}$ dependent compaction in molecular dimensions was observed. Most interestingly, a direct correlation between $\mathrm{pH}$ induced modulation of functional activity and orientation of Trp 101 present in the active site of the enzyme was observed. Sodium halides nullified the $\mathrm{pH}$ induced global changes in the enzyme, however differential effect of these salts on the active site microenvironment and functional activity of the enzyme was observed.

Conclusions: The study unequivocally demonstrates that $\mathrm{pH}$ induced selective modification of active site microenvironment and not global change in structure or oligomeric status of the enzyme is responsible for the $\mathrm{pH}$ dependent change in enzymatic activity of PSAT.
\end{abstract}

\section{Background}

PSAT is a vitamin $B_{6}$-dependent enzyme that belongs to the $\alpha$-family of pyridoxal-5'-phosphate (PLP) enzymes. It catalyzes the reversible conversion of 3-phosphohydroxypyruvate to L-phosphoserine in a glutamate linked transamination reaction, the second step of phosphorylated serine biosynthetic pathway. Earlier studies on PSAT from E coli [1], Bacillus alcalophilus [2], Arabidopsis thaliana [3], and Homo sapiens [4] suggests that the enzyme exist as a homodimer with a subunit molecular mass between 40 to $48 \mathrm{kDa}$. Each subunit is predominantly composed of two domains, a large PLP binding domain and a small domain comprising of $\mathrm{C}$ terminal part along with a short $\mathrm{N}$-terminal portion [1].

\footnotetext{
* Correspondence: bhakuniv@rediffmail.com
'Division of Molecular and Structural Biology, Central Drug Research Institute,

* Correspondence: bhakuniv@rediffmail.com
'Division of Molecular and Structural Biology, Central Drug Research Institute, Chattar Manzil Palace, Council of scientific and industrial research (CSIR), Lucknow 226001, India
}

Structurally PSAT is a $\alpha / \beta$ protein with one PLP molecule present in the active site, per monomer. The dimeric configuration of the enzyme is essential for the functional activity [5]. The active site amino acid residues are nearly conserved in all PSATs (Fig. 1A).

Entamoeba histolytica is a protozoan parasite that infects the gastrointestinal tract and causes amoebic colitis and extra intestinal abscesses in humans [6]. Role of L- serine in a number of important metabolic pathways in the parasite has been well established $[7,8]$ Predominantly it serves as a precursor molecule for Lcysteine biosynthesis which plays important role in survival, growth, attachment $[9,10]$, anti-oxidative defense [11], and Fe-S cluster biosynthesis [12]. From the amino acid sequence alignment and phylogenetic analyses EhPSAT shows close association with bacteroide PSAT [13]. 

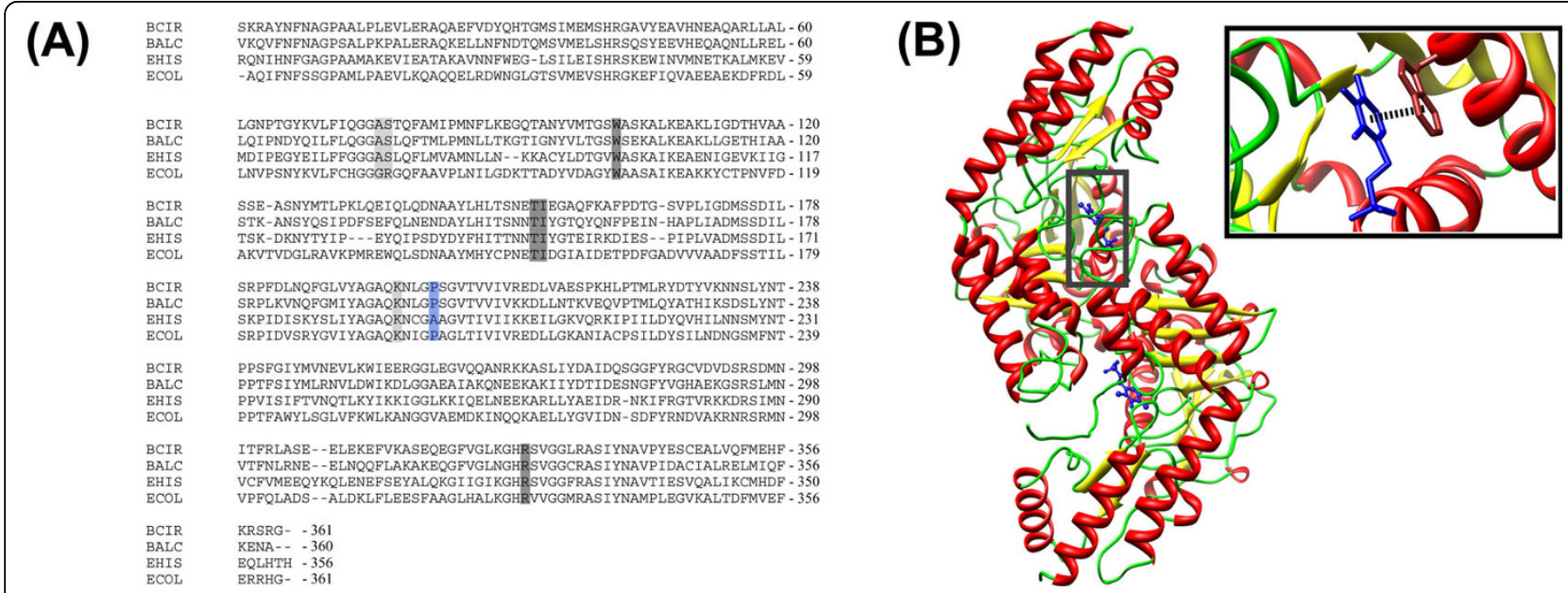

Figure 1 (A) Alignment of the amino acid sequence of PSAT from Bacillus circulans spp. Alkalophilus (BCIR), Bacillus alcalophilus (BALC), Entamoeba histolytica (EHIS), and E coli (ECOL). Highlighted letters indicate active site residues that interact with the cofactor PLP (light grey) and $\mathrm{Cl}^{-}$ions (dark grey). The substitution of Pro201 (BCIR PSAT) to Ala195 (EhPSAT) in the loop region has been highlighted in blue. (B) Homology model of EhPSAT dimer visualized through UCSF chimera. The cofactor PLP present in the active site is represented in cyan color. The inset shows stacking of the cofactor PLP(cyan color) and the Trp101(brown color) at the active site.

The cofactor PLP plays a significant role in structural stabilization of PSAT from extremophiles [14] and serves as a vital tool for understanding the active site stochiometry and conformational rearrangements under various experimental conditions. We have carried out a detailed study on the $\mathrm{pH}$ induced changes in the functional, structural and stability properties of EhPSAT and effect of salts on these changes. Additional file 1 carries detailed information regarding the materials and experimental procedures applied in the presented study.

\section{Results}

\section{EhPSAT production and oligomeric state}

The purified protein was homogenous as indicated by a single protein band on SDS-PAGE (Fig. 2A) and a single peak in ESI-MS of molecular mass about $43 \mathrm{kDa}$ (data not shown). The SEC profile of EhPSAT (Fig. 3A) along with glutaraldehyde crosslinking experiments (Fig. 3B) demonstrates that the recombinant EhPSAT is a homodimer under physiological conditions.

\section{$\mathrm{pH}$ dependent changes in functional and structural properties of EhPSAT \\ Functional property}

EhPSAT showed $\mathrm{pH}$ dependent bell shaped enzymatic activity profile for forward reaction with maximum activity at pH 8.5 (Fig. 2B). At pH 10 and 6.0, the enzyme retained about $50 \%$ and $20 \%$ residual activity, respectively.

For understanding the structural basis of such a characteristic $\mathrm{pH}$ dependence of enzymatic activity, structural changes in the enzyme under these conditions, were studied.

\section{Secondary structure}

The secondary structure elements are generally conserved among PSAT family of enzymes $[1,2]$. The secondary structure of EhPSAT as characterized by far-UV $C D$ shows that it is a $\alpha / \beta$ type protein (Fig. $2 \mathrm{C}$ inset). No significant alterations in the CD signal at $222 \mathrm{~nm}$ were observed for the enzyme between $\mathrm{pH} 9$ and 6(Fig. $2 \mathrm{C})$. Hence, $\mathrm{pH}$ change does not significantly affect the secondary structure of EhPSAT.

\section{Active site microenvironment}

No significant alteration in fluorescence polarization of PLP was observed between pH 6 to 9 suggesting no change in orientation of cofactor PLP of EhPSAT with change in $\mathrm{pH}$ (Fig. 2D). Interestingly for tryptophan residues, with decrease in $\mathrm{pH}$ from 10 to 6 an almost linear enhancement in fluorescence polarization was observed, (Fig. 2E). This demonstrates that low $\mathrm{pH}$ induces restriction in orientation of tryptophan moiety present in enzyme. EhPSAT contains 3 tryptophan residues, two in the N-terminal region and one, Trp 101, in the active site. We wanted to see whether the above observed changes correspond to the active site tryptophan moiety or not.

On reduction of PLP aldimine, the cofactor serves as a reporter molecule itself and also as an energy acceptor of tryptophan fluorescence provided the two moieties are within a distance of $5 \AA[15,16]$. For EhPSAT, PLP and Trp 101 are stacked close to each other at the active site (Fig. 1B inset). Fig. 2F summarizes the effect of $\mathrm{pH}$ on the fluorescence spectra of EhPSAT on excitation at $295 \mathrm{~nm}$. At $\mathrm{pH} 8.0$ and 9.0, single fluorescence emission maxima at $335 \mathrm{~nm}$ and $340 \mathrm{~nm}$, respectively 


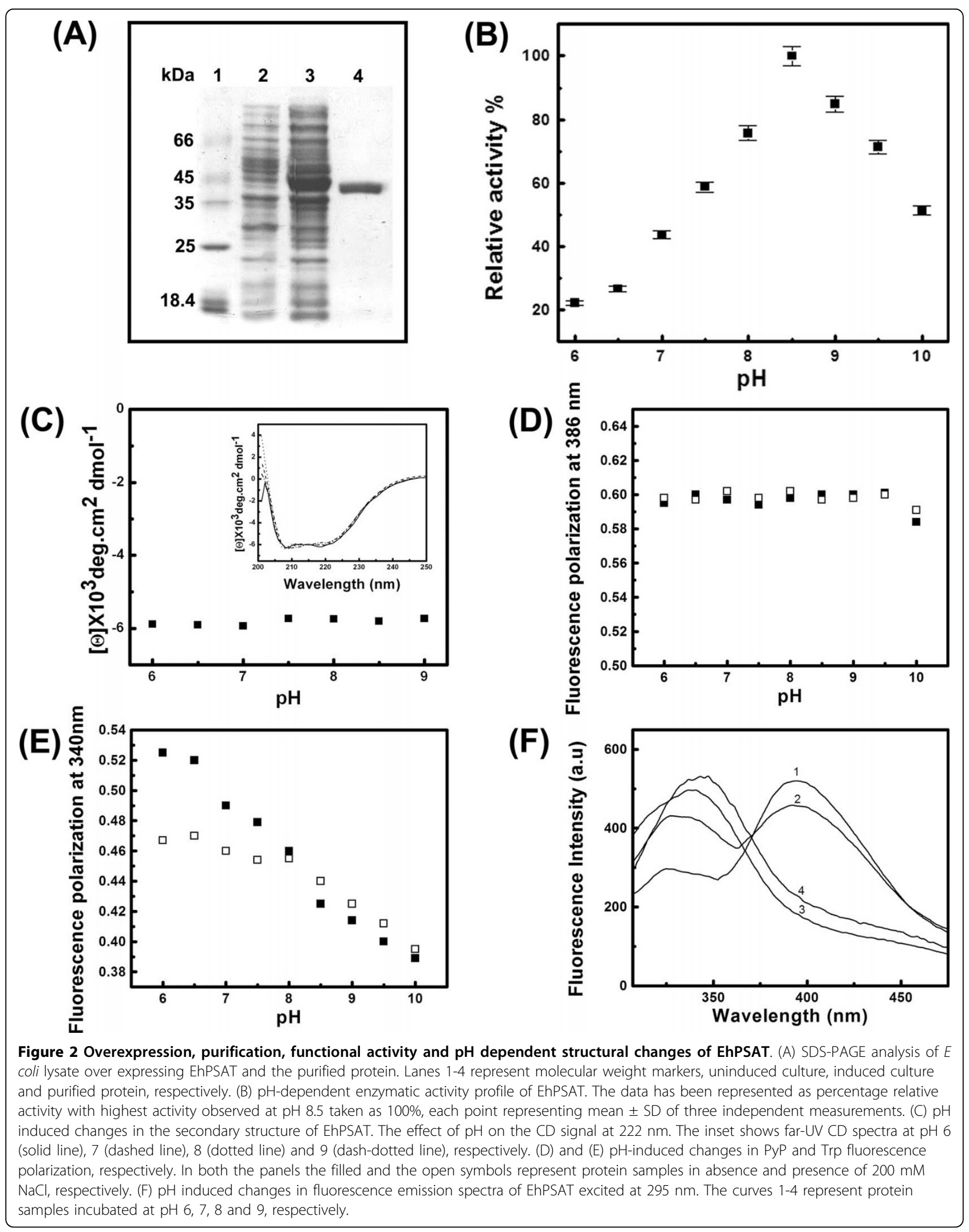



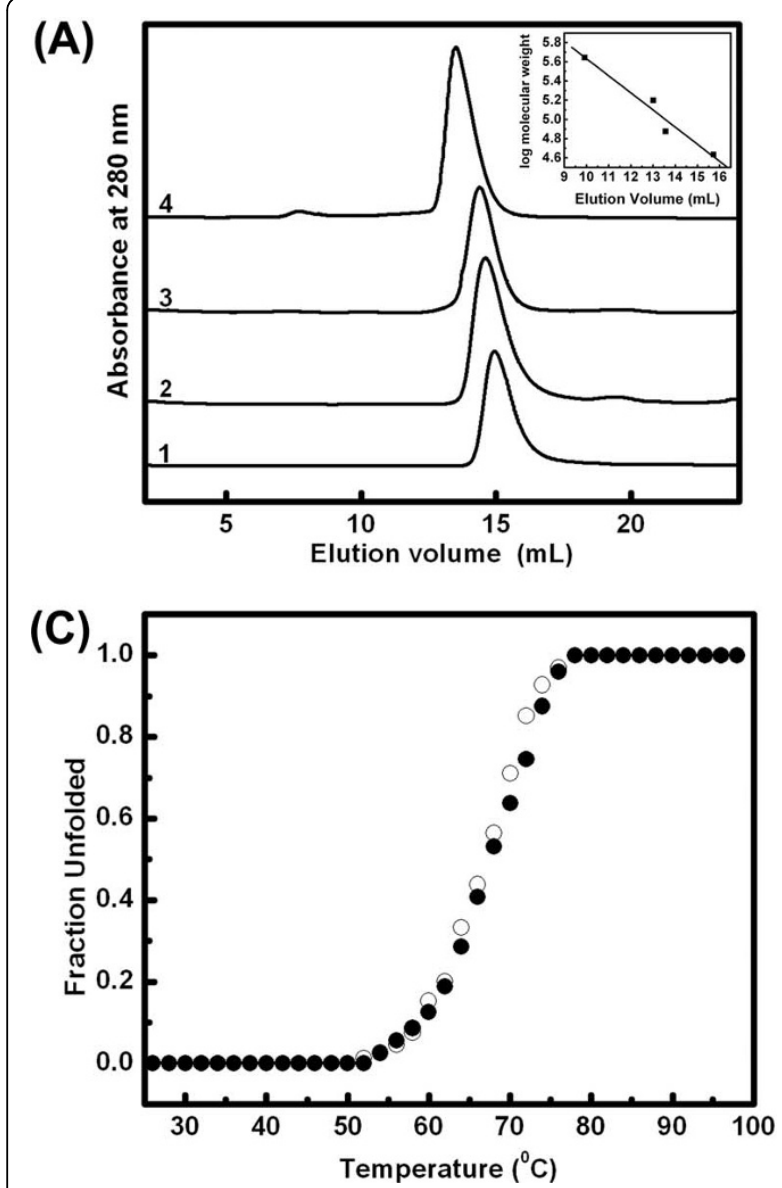

(B)
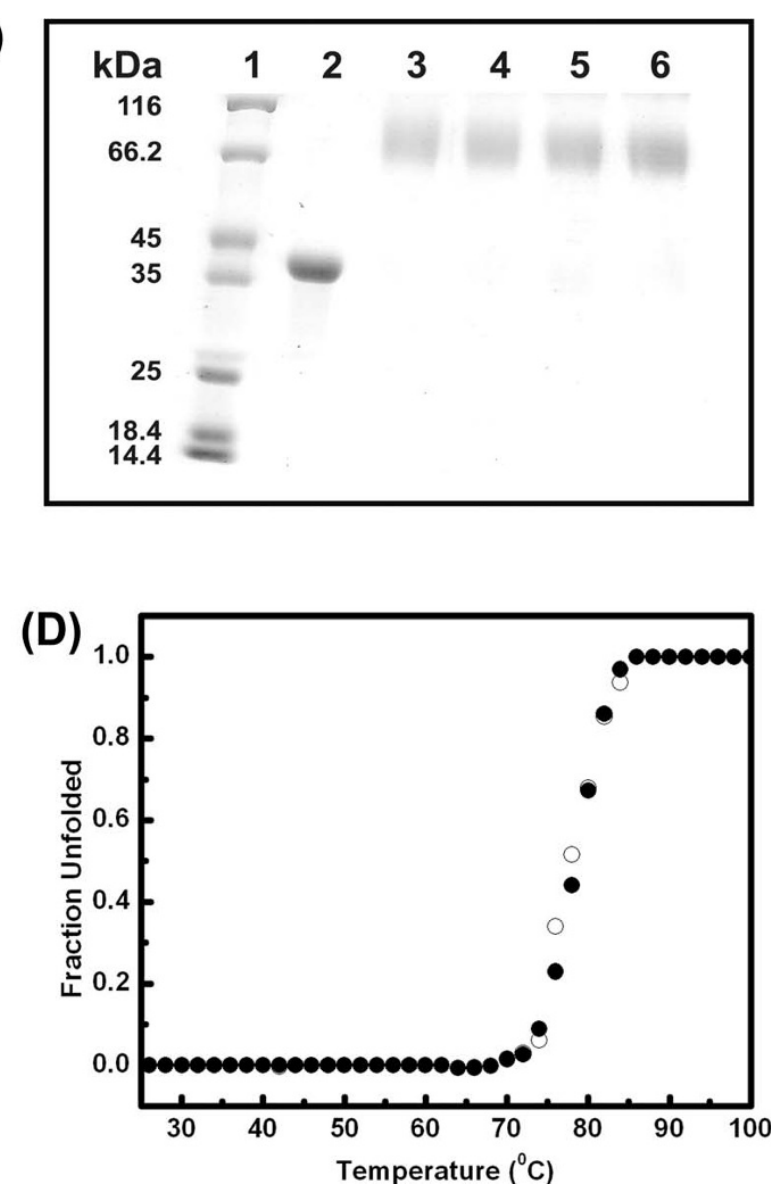

Figure $3 \mathrm{pH}$ induced compaction of EhPSAT, oligomeric state and thermal stability. (A) Curves 1-4 represent SEC profile of EhPSAT on a superdex 200, 10/300 GL column at pH 6, 7, 8 and 9, respectively. The inset shows the graph of elution volume plotted against standard molecular mass markers. The proteins are (1) $440 \mathrm{KDa}$ (ferritin), (2)158 KDa (aldolase), (3) $75 \mathrm{KDa}$ (conalbumin) and (4) $43 \mathrm{KDa}$ (ovalbumin). (B) 10\% SDS-PAGE profile of glutaraldehyde crosslinked sample of EhPSAT incubated at different pH. Lane 1-6 represent molecular weight markers, uncrosslinked EhPSAT, and glutaraldehyde crosslinked form of EhPSAT at pH 9, 8, 7 and 6, respectively. Cooperative thermal unfolding of EhPSAT at pH 8.5 (panel C) and 6 (panel D) as measured by loss of CD ellepticity at $222 \mathrm{~nm}$ and $415 \mathrm{~nm}$. A linear extrapolation of baselines in pre and post transition regions was used to determine the fraction unfolded protein within the transition region by assuming a two state mechanism of unfolding. The open and filled circle represent for far and near UV-CD signals, respectively. The thermal transition of the enzyme was found to be irreversible with precipitation observed at the end of the scan.

was observed. However, at pH 6 and 7, two clear emission maxima centered at 386 and $335 \mathrm{~nm}$, respectively were observed. Furthermore, on decrease in $\mathrm{pH}$ from 9 to 6 , a decrease in intensity of signal at $335 \mathrm{~nm}$ and a concomitant increase in intensity of signal at $386 \mathrm{~nm}$ were observed. These observations demonstrate that between pH 9 and 6 the two fluorophores PLP and Trp101 come closer to each other and show FRET. The FRET studies along with the PLP polarization studies (Fig. 2D to $2 \mathrm{~F}$ ) demonstrate that with change in $\mathrm{pH}$, the orientation of Trp101 residue is modified such that it comes close to PLP cofactor in 3D space.

Molecular dimension, Subunit configuration and stability On decrease in $\mathrm{pH}$ from 9 to 6 , decrease in the hydrodynamic radii of the enzyme (Fig. 3A) was observed indicating either $\mathrm{pH}$-induced dissociation of native dimer to monomer or compaction of dimeric conformation. Chemical crosslinking is a well accepted technique for studying changes in the oligomeric status of a protein under experimental conditions [16-21]. Fig. 3B shows the SDS PAGE profile of the glutaraldehyde cross linked protein samples. A single species corresponding to dimer of enzyme was observed under all the conditions studied. The stability of dimeric configuration over a broad $\mathrm{pH}$ range has also been reported for PSAT from Bacillus circulans ssp. Alkalophilus. These studies collectively demonstrate that PSAT dimer is stable over a broad $\mathrm{pH}$ range.

Fig. 3C and 3D shows thermal denaturation profile of EhPSAT at pH 8.5 and 6 as monitored by the loss of CD signal at 222 and $415 \mathrm{~nm}$. Superimposable far-UV 
and near UV CD signals demonstrate a high degree of structural cooperativity exist in EhPSAT under these conditions. However, for $\mathrm{pH} 8.5$ and $6, T_{\mathrm{m}}$ of about $67^{\circ}$ $\mathrm{C}$ and $79^{\circ} \mathrm{C}$, respectively were observed. These obeservations are in agreement with the ProTherm database as reported earlier for Bacillus circulans ssp. Alkalophilus [14]. Hence, decrease in $\mathrm{pH}$ leads to enhanced thermal stability of PSAT.

\section{Effect of sodium halides on the enzymatic activity}

Salts affect the physico-chemical properties of proteins primarily through modulation of electrostatic and hydrophobic interactions acting on the protein molecule. Fig. 4A shows the effect of $200 \mathrm{mM} \mathrm{NaF}, \mathrm{NaCl}$ and $\mathrm{NaBr}$ on the enzymatic activity at $\mathrm{pH} 8.5 . \mathrm{NaCl}$ and $\mathrm{NaBr}$ inhibited the enzymatic activity in a concentration dependent manner, while NaF showed no such effect. Detailed studies with $\mathrm{NaCl}$ and $\mathrm{NaF}$ on $\mathrm{pH}$ induced changes in the functional activity (Fig. 4B) clearly show no significant change in the $\mathrm{pH}$ dependence of enzymatic activity for $\mathrm{NaF}$. Interestingly, in presence of $\mathrm{NaCl}$ the maximum activity was found to be retained over a broader range of $\mathrm{pH} 7.5$ to 8.5 and even at $\mathrm{pH} 6$ about $40 \%$ residual activity was observed.

Effect of $\mathrm{NaCl}$ and $\mathrm{NaF}$ on $\mathrm{pH}$ induced modification at the active site and strain of internal aldimine of EhPSAT

With change in $\mathrm{pH}$ from 8.5 to 6 in the presence of $\mathrm{NaCl}$, no effect on fluorescence polarization of PLP was observed (Fig. 2D). However, the tryptophan fluorescence polarization was found to enhance under these conditions, but to a significantly lower extent as compared to that in absence of $\mathrm{NaCl}$. This demonstrates that the $\mathrm{pH}$-induced change in orientation of tryptophan residue is modulated by the presence of $\mathrm{NaCl}$ (Fig. 2E). FRET analyses also support these observations, Fig. 4C and $4 \mathrm{D}$ show no significant alteration in the tryptophan microenvironment in presence of $\mathrm{NaCl}$ between $\mathrm{pH} 9$ and 6. In contrast for NaF, FRET profile similar to that in absence of salt was observed (Fig. 2F).

The first step in the catalytic mechanism of the enzyme is transfer of a proton of charged substrate amino group to the unprotonated imine nitrogen of the internal Shiff's base in the Michaelis complex. This is followed by nucleophilic attack of the substrate amino group on C4' carbon atom of the cofactor resulting in formation of external aldimine intermediate between PLP and substrate. In PLP catalysis, the electron sink property of cofactor plays an important role. It has been proposed that in the catalytic mechanism the strain and distortion of conjugated $\pi$-electron system of PLP and internal aldimine in PSAT are important [22]. When the internal aldimine is protonated a single positive CD band is observed at $410-415 \mathrm{~nm}$ but it is shifted to 345 $350 \mathrm{~nm}$ when the internal aldimine is deprotonated [22]. The additional file 2 shows the $\mathrm{pH}$ dependence of
(A)

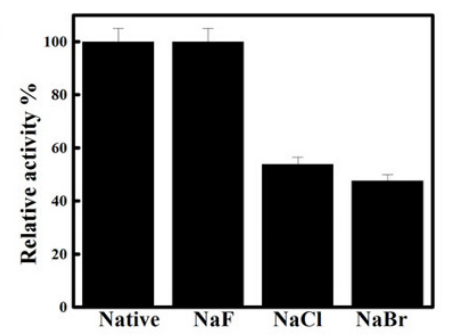

(D)

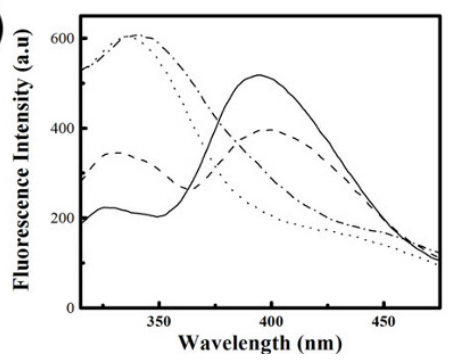

(B)

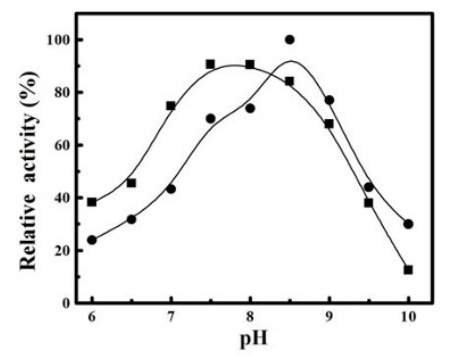

(E)

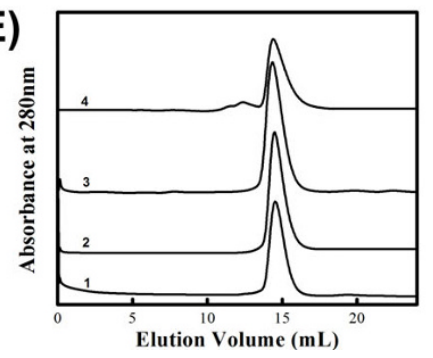

(C)

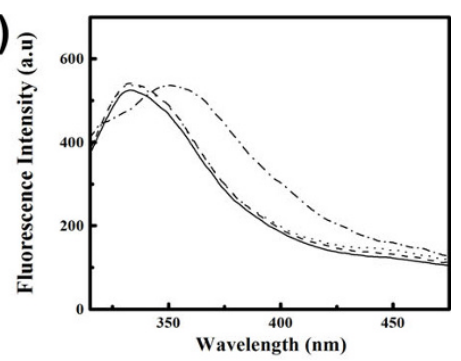

(F)

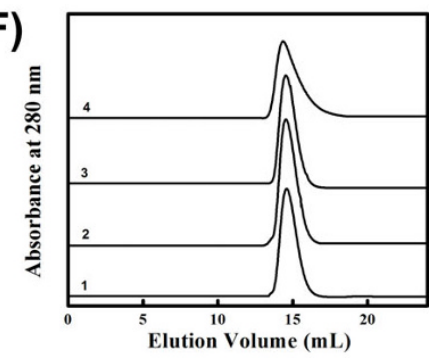

Figure 4 Effect of Sodium halides on pH dependent changes in functional activity and structural features of EhPSAT. (A) Inhibition of functional activity in presence of $200 \mathrm{mM}$ concentration of various Sodium halides. The activity of EhPSAT in absence of salt (native protein) at $\mathrm{pH} 8.5$ was taken as 100\%. (B) pH dependent enzymatic activity profile of EhPSAT in presence of $200 \mathrm{mM}$ concentration of NaCl (rectangles) and $\mathrm{NaF}$ (circles), respectively. For samples in presence of $\mathrm{NaCl}$ and $\mathrm{NaF}$ the highest value observed was taken as $100 \%$. Fluorescence emission spectra of EhPSAT in presence of $200 \mathrm{mM} \mathrm{NaCl}$ (Panel C) and $\mathrm{NaF}$ (Panel D) at different pH. The different curves in both the panel represent protein samples at pH 6 (solid line), 7 (dashed line), 8 (dotted line) and 9 (dash dotted line). SEC profile at various pH in presence of $\mathrm{NaCl}$ (panel E) and NaF (panel F). The curves 1-4 represent SEC profile in presence of $200 \mathrm{mM}$ concentration of salts at $\mathrm{pH} 6,7$ and 8 , and 9 respectively. 
near-UV CD spectra of EhPSAT in presence and absence of $\mathrm{NaCl}$. Under both conditions, between $\mathrm{pH} 6$ and 8 , a major CD band at 410-415 $\mathrm{nm}$ was observed suggesting that the protein is predominantly in protonated state under these conditions. However, for protein samples at $\mathrm{pH} 9$ and 10 the major $\mathrm{CD}$ band is shifted to $345-350 \mathrm{~nm}$ suggesting that the protein is predominantly in unprotonated state. These observations indicate that the $\mathrm{pH}$ - induced difference in the functional activity in absence and presence of $\mathrm{NaCl}$ is not due to modulation of $\mathrm{pKa}$ of the catalytic reaction.

Effect of $\mathrm{NaCl}$ and NAF on pH-induced compaction and thermal unfolding of EhPSAT

Fig. $4 \mathrm{E}$ and $4 \mathrm{~F}$, presents the SEC profile of EhPSAT at $\mathrm{pH} 6,7,8$ and 9 in presence of $200 \mathrm{mM} \mathrm{NaCl}$ and $\mathrm{NaF}$, respectively. No significant change in molecular dimension of the protein with change in $\mathrm{pH}$ was observed suggesting that $\mathrm{pH}$ induced compaction is abolished by salts. Fig. $5 \mathrm{~A}$ to $5 \mathrm{D}$, shows the thermal unfolding at $\mathrm{pH}$ 8.5 and 6.0 , respectively in presence of $200 \mathrm{mM} \mathrm{NaCl}$ and NaF. At pH 8.5, a significant difference in Tm associated with loss of secondary structure and dissociation of PLP from enzyme was observed suggesting the thermal denaturation process in presence of salts to be a noncooperative event. However, at $\mathrm{pH} 6.0$ in presence of salts changes similar to that in absence of salts were observed.

\section{Discussion}

The active site of PSAT is optimized for binding of Lglutamate, 3-phosphohydroxy pyruvate, 2-oxoglutarate and L-phosphoserine [1]. Structural fluctuations in the native state of protein significantly influence the functional activity [23]. The secondary structure of EhPSAT is resistant to $\mathrm{pH}$ change between 9 and 6 similar to
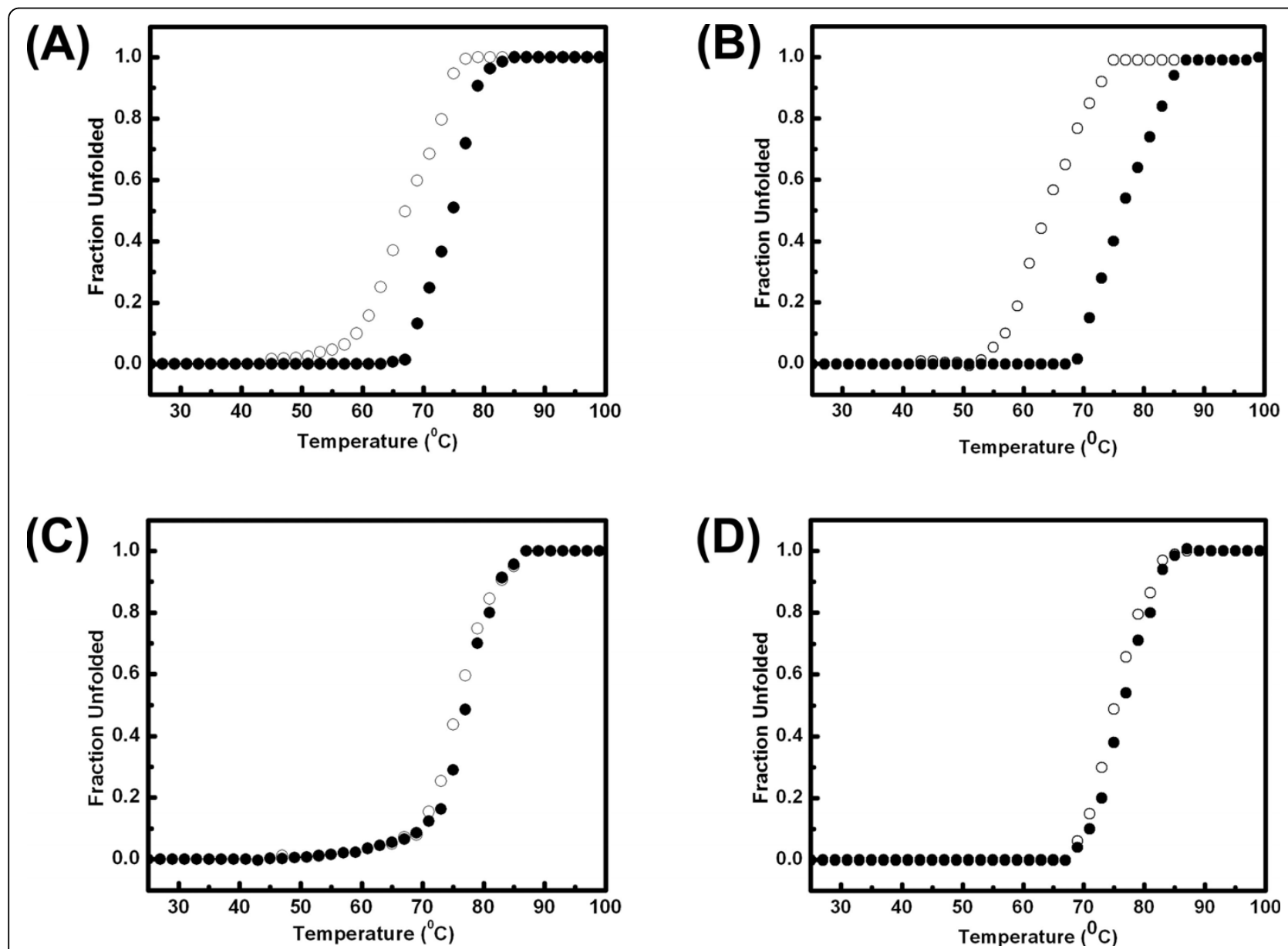

Figure 5 Thermal unfolding in presence of Sodium halides. Thermal denaturation profile at pH 8.5 in presence of $200 \mathrm{mM} \mathrm{NaCl}$ (panel A), $\mathrm{NaF}$ (panel B) and at pH 6 in presence of $200 \mathrm{mM} \mathrm{NaCl}$ (panel C) and $\mathrm{NaF}$ (panel D), respectively. The open and closed circles represent CD ellepticity measured at $222 \mathrm{~nm}$ and $415 \mathrm{~nm}$, respectively. A linear extrapolation of baselines in pre and post transition regions was used to determine the fraction unfolded protein within the transition region by assuming a two state mechanism of unfolding. The thermal transition of the enzyme was found to be irreversible with precipitation observed at the end of the scan. 
Bacillus circulans spp. Alkalophilus (BCIR) PSAT [14]. Hence, the secondary structure of PSATs is stable over a wide $\mathrm{pH}$ range and so does not play any significant role in modulation of enzymatic activity.

Crystallographic studies on BALC PSAT clearly suggest the presence of ion binding sites on PSAT molecule[2]. Four $\mathrm{Cl}^{-}$ions in particular are found to be present 2 per monomer close to the active site residue Trp102. Main chain amides of Ser101, Trp102, Thr152 and Ile153 form the binding site for one $\mathrm{Cl}^{-}$ion and the second one is located between positively charged side chain of Arg334 and the $\mathrm{N}^{\varepsilon 1}$ atom of Trp102(amino acid position described according to BALC PSAT) [2]. Amino acids responsible for chloride ion binding are conserved in BALC PSAT and EhPSAT (Fig. 1A). It is possible that direct binding of $\mathrm{Cl}^{-}$and $\mathrm{Br}^{-}$ions (ionic radii $1.67 \AA$ and $1.96 \AA$, respectively) in the active site leads to structural modifications which results in significant loss of functional activity. $\mathrm{F}^{-}$Ions being smaller in size (ionic radii $1.36 \AA$ ) will not bind to the anion binding site(s) with similar affinity and hence not bring about similar change in active site conformation as chloride or bromide ions. This possibility is also supported by the observation that $\mathrm{SO}_{4}{ }^{2-}$ ions as they also inhibit the enzymatic activity similar to chloride or bromide ions (Data not shown).

The two active sites of PSAT dimer are situated approximately $20 \AA$ apart at the subunit interface. In each active site a PLP molecule is bound with an aldimine linkage to Lys 191 and aditionally hydrogen bonded to residues from the two large domains, besides this prominent stacking interaction at the active site between the pyridine ring of PLP and the indole ring of Trp101, which are usually separated by a distance of approximately $5 \AA$, occurs on the re-face of the cofactor [[14], this study]. No change in orientation of PLP and tryptophan aromatic ring occurs upon $\mathrm{pH}$ change [14]. In case of EhPSAT we observed a pH dependent change in orientation of tryptophan moiety but not for the PLP as monitored by fluorescence polarization (Fig. 2D and $2 \mathrm{E}$ ). Presence of two fluorophores predominantly stacked with each other in the active site invariably serves as an important tool for probing fine fluctuations of the $\mathrm{pH}$ sensitive active site microenvironment. On moving towards low $\mathrm{pH}$ (from 8 to 6), the enzyme starts to gradually loose its activity and under these conditions there is a gradual appearance of FRET, suggesting an inwards movement of tryptophan moiety thus bringing it close to PLP in space. In contrast, on moving towards basic $\mathrm{pH}$ i.e. from 8 towards 10 , a shift of tryptophan fluorescence emission maximum from $335 \mathrm{~nm}$ to 340 $\mathrm{nm}$ was observed, which shows an opening of native conformation of enzyme with partial solvent exposure of tryptophan residue(s). Such $\mathrm{pH}$ dependent changes in the active site dynamics suggest relaxation (open) and compaction (close) of active site conformation. Such a dynamic movement in the active site would interfere with proper docking of substrates and responsible for loss of enzymatic activity. Support to such a possibility comes from the $\mathrm{pH}$ studies in presence of $\mathrm{NaCl}$ where no relative movement of the two fluorophores with $\mathrm{pH}$ change (as discussed in paper) was observed and under these conditions no significant alteration in the enzymatic activity was observed over a wider $\mathrm{pH}$ range. Conformational changes observed in the present study have not been reported earlier based on the comparison of crystal structures of PSAT at $\mathrm{pH} 4.6$ and $\mathrm{pH}$ 8.5[14] because of the fact that protein crystal packing may interfere with fine conformational changes observed in the solution $[24,25]$. These differences in the active site microenvironment between EhPSAT and BCIR PSAT might probably be due to change in flexibility of the loop region 199-202 which is located near the active site. In this loop the Pro201 in BCIR PSAT is changed to Ala195 in EhPSAT (shown in Fig. 1). Such a change will definitely bring about differences in the flexibility of the loop and subsequently modulate the packing of the protein in the vicinity of active site. The studies presented in the paper clearly demonstrate that local changes at active site microenvironment and not global changes in the protein conformation are responsible for the $\mathrm{pH}$ induced modulation of functional activity of PSAT.

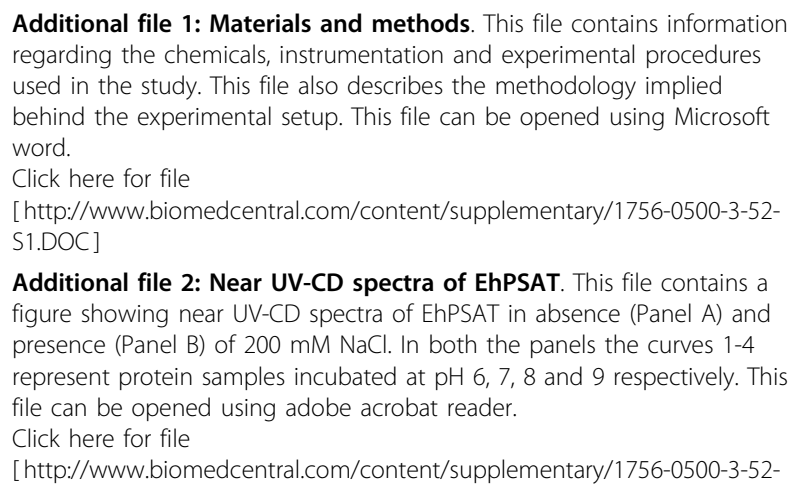

Additional file 2: Near UV-CD spectra of EhPSAT. This file contains a figure showing near UV-CD spectra of EhPSAT in absence (Panel A) and presence (Panel B) of $200 \mathrm{mM} \mathrm{NaCl}$. In both the panels the curves 1-4 represent protein samples incubated at $\mathrm{pH} 6,7,8$ and 9 respectively. This file can be opened using adobe acrobat reader. Click here for file

[http://www.biomedcentral.com/content/supplementary/1756-0500-3-52S2.PDF ]

\section{Abbreviations}

PSAT: phosphoserine aminotransferase; PLP: pyridoxal-5'-phosphate; Ni-NTA: nickel nitrilotriacetic acid; HPAP: hydroxypyruvic acid phosphate(also called phosphohydroxy pyruvate); SEC: size exclusion chromatography; FRET: fluorescence resonance energy transfer; ESI-MS: electrospray ionization mass spectroscopy.

\section{Acknowledgements}

VM wishes to thank Council of scientific and industrial research (CSIR), New Delhi, for financial assistance. Ashutosh kumar is acknowledged for bioinformatics analysis. Funding for article processing charges: Annual S S 
Bhatnagar award fund (to VB). This is communication no.7649 from CDRI, Lucknow (India).

\section{Author details}

'Division of Molecular and Structural Biology, Central Drug Research Institute, Chattar Manzil Palace, Council of scientific and industrial research (CSIR), Lucknow 226001, India. ${ }^{2}$ Department of Biochemistry, Rajendra Memorial Research Institute of Medical Sciences, AgamKuan, Patna 800007, India. ${ }^{3}$ Department of Parasitology, National Institute of Infectious diseases, 1-23-1 Toyama, Shinjuku-Ku, Tokyo 162-8640, Japan.

\section{Authors' contributions}

VM carried out all the experiments and data analysis. VB concieved and directed the study and was responsible for final evaluation of results. VM and VB drafted the manuscript. VA and TN provided the clone of PSAT and were part of the discussions during drafting of the manuscript. All authors read and approved the final manuscript.

\section{Competing interests}

The authors declare that they have no competing interests.

Received: 3 August 2009 Accepted: 3 March 2010

Published: 3 March 2010

\section{References}

1. Hester G, Stark W, Moser M, Kallen J, Markovic-Housley Z, Jansonius JN: Crystal structure of phosphoserine aminotransferase from Escherichia coli at $2.3 \mathrm{~A}$ resolution: comparison of the unligated enzyme and a complex with alpha-methyl-I-glutamate. J Mol Biol 1999, 286(3):829-850.

2. Dubnovitsky AP, Kapetaniou EG, Papageorgiou AC: Enzyme adaptation to alkaline $\mathrm{pH}$ : atomic resolution $(1.08 \mathrm{~A})$ structure of phosphoserine aminotransferase from Bacillus alcalophilus. Protein Sci 2005, 14:97-110.

3. Ho CL, Noji M, Saito M, Yamazaki M, Saito K: Molecular characterization of plastidic phosphoserine aminotransferase in serine biosynthesis from Arabidopsis. Plant J 1998, 16:443-52.

4. Baek JY, Jun DY, Taub D, Kim YH: Characterization of human phosphoserine aminotransferase involved in the phosphorylated pathway of L-serine biosynthesis. Biochem J 2003, 373:191-200.

5. John RA: Pyridoxal phosphate-dependent enzymes. Biochim Biophys Acto 1995, 1248:81-96.

6. Ali V, Nozaki T: Current therapeutics, their problems, and sulfurcontaining-amino-acid metabolism as a novel target against infections by "amitochondriate" protozoan parasites. Clin Microbiol Rev 2007, 20:164-87.

7. Ali V, Hashimoto T, Shigeta Y, Nozaki T: Molecular and biochemical characterization of D-phosphoglycerate dehydrogenase from Entamoeba histolytica. A unique enteric protozoan parasite that possesses both phosphorylated and nonphosphorylated serine metabolic pathways. Eur J Biochem 2004, 271:2670-81.

8. Ali V, Shigeta Y, Nozaki T: Molecular and structural characterization of NADPH-dependent d-glycerate dehydrogenase from the enteric parasitic protist Entamoeba histolytica. Biochem J 2003, 375:729-36.

9. Gillin FD, Diamond LS: Attachment of Entamoeba histolytica to glass in a defined maintenance medium: specific requirement for cysteine and ascorbic acid. J Protozool 1980, 27:474-8.

10. Gillin FD, Diamond LS: Entamoeba histolytica and Giardia lamblia: growth responses to reducing agents. Exp Parasitol 1981, 51:382-91.

11. Nozaki T, Asai T, Sanchez LB, Kobayashi S, Nakazawa M, Takeuchi T: Characterization of the gene encoding serine acetyltransferase, a regulated enzyme of cysteine biosynthesis from the protist parasites Entamoeba histolytica and Entamoeba dispar. Regulation and possible function of the cysteine biosynthetic pathway in Entamoeba. J Biol Chem 1999, 274:32445-52.

12. Ali V, Shigeta Y, Tokumoto U, Takahashi Y, Nozaki T: An intestinal parasitic protist, Entamoeba histolytica, possesses a non-redundant nitrogen fixation-like system for iron-sulfur cluster assembly under anaerobic conditions. J Biol Chem 2004, 279:16863-74.

13. Ali V, Nozaki T: Biochemical and functional characterization of phosphoserine aminotransferase from Entamoeba histolytica, which possesses both phosphorylated and non-phosphorylated serine metabolic pathways. Mol Biochem Parasitol 2006, 145:71-83.
14. Kapetaniou EG, Thanassoulas A, Dubnovitsky AP, Nounesis G, Papageorgiou AC: Effect of $\mathrm{pH}$ on the structure and stability of Bacillus circulans ssp. alkalophilus phosphoserine aminotransferase: thermodynamic and crystallographic studies. Proteins 2006, 63:742-53.

15. Cai K, Schirch V: Structural studies on folding intermediates of serine hydroxymethyltransferase using fluorescence resonance energy transfer. J Biol Chem 1996, 271:27311-20.

16. Chaturvedi S, Bhakuni V: Unusual structural, functional, and stability properties of serine hydroxymethyltransferase from Mycobacterium tuberculosis. J Biol Chem 2003, 278:40793-805.

17. Mishra P, Akhtar MS, Bhakuni V: Unusual structural features of the bacteriophage-associated hyaluronate lyase (hylp2). J Biol Chem 2006, 281:7143-50

18. Bhatt AN, Khan MY, Bhakuni V: The C-terminal domain of dimeric serine hydroxymethyltransferase plays a key role in stabilization of the quaternary structure and cooperative unfolding of protein: domain swapping studies with enzymes having high sequence identity. Protein Sci 2004, 13:2184-95.

19. Akhtar MS, Ahmad A, Bhakuni V: Divalent cation induced changes in structural properties of the dimeric enzyme glucose oxidase: dual effect of dimer stabilization and dissociation with loss of cooperative interactions in enzyme monomer. Biochemistry 2002, 41:7142-9.

20. Akhtar MS, Ahmad A, Bhakuni V: Guanidinium chloride- and urea-induced unfolding of the dimeric enzyme glucose oxidase. Biochemistry 2002, 41:3819-27.

21. Singh K, Bhakuni V: Cation induced differential effect on structural and functional properties of Mycobacterium tuberculosis alphaisopropylmalate synthase. BMC Struct Biol 2007, 7:39.

22. Dubnovitsky AP, Ravelli RB, Popov AN, Papageorgiou AC: Strain relief at the active site of phosphoserine aminotransferase induced by radiation damage. Protein Sci 2005, 14:1498-507.

23. Singh K, Bhakuni V: Toxoplasma gondii ferredoxin-NADP+ reductase: Role of ionic interactions in stabilization of native conformation and structural cooperativity. Proteins 2008, 71:1879-88.

24. Kapoor P, Sahasrabuddhe AA, Kumar A, Mitra K, Siddigi MI, Gupta CM: An unconventional form of actin in protozoan hemoflagellate, Leishmania. Biol Chem 2008, 283:22760-73.

25. Rould MA, Wan Q, Joel PB, Lowey S, Trybus KM: Crystal structures of expressed non-polymerizable monomeric actin in the ADP and ATP states. J Biol Chem 2006, 281:31909-19.

doi:10.1186/1756-0500-3-52

Cite this article as: Mishra et al: Entamoeba histolytica Phosphoserine aminotransferase (EhPSAT): insights into the structure-function relationship. BMC Research Notes 2010 3:52.

\section{Submit your next manuscript to BioMed Central and take full advantage of:}

- Convenient online submission

- Thorough peer review

- No space constraints or color figure charges

- Immediate publication on acceptance

- Inclusion in PubMed, CAS, Scopus and Google Scholar

- Research which is freely available for redistribution
Biomed Central 